\title{
Concentration urinaire de bisphénol $A$ et obésité chez les adultes : résultats de l'Enquête canadienne sur les mesures de la santé
}

\author{
Minh T. Do, Ph. D. (1,2,3); Vicky C. Chang, M.P.H. (1,2); Michelle A. Mendez, Ph. D. (4); Margaret de Groh, Ph. D. (1)
}

Cet article a fait l'objet d'une évaluation par les pairs.

\section{Résumé}

Introduction : Des études chez l'animal ont révélé que l'exposition au bisphénol A (BPA) affecte le métabolisme des lipides et favorise la prise de poids. Des études épidémiologiques récentes appuient aussi l'existence d'un lien entre le BPA et l'obésité chez l'humain, mais la plupart d'entre elles se limitent à une seule mesure de l'adiposité ou ne tiennent pas compte des facteurs de confusion alimentaires possibles. Cette étude vise à examiner les associations entre les concentrations urinaires de BPA et les mesures de l'adiposité dans un échantillon national représentatif des adultes canadiens.

Méthodologie : Nous avons réalisé des analyses à l'aide de données de biosurveillance et de données anthropométriques mesurées directement auprès de 4733 adultes de 18 à 79 ans dans le cadre de l'Enquête canadienne sur les mesures de la santé (2007-2011). Nous avons utilisé des modèles de régression logistique multinomiale et binaire pour estimer les associations entre, d'une part, les concentrations urinaires de BPA et, d'autre part, les diverses catégories d'indice de masse corporelle (IMC) (embonpoint par opposition à poids insuffisant ou normal; obésité par opposition à poids insuffisant ou normal) et un tour de taille élevé (102 cm ou plus pour les hommes; $88 \mathrm{~cm}$ ou plus pour les femmes) en tenant compte des facteurs de confusion possibles. Des analyses de régression linéaire ont aussi été effectuées pour évaluer les associations entre les concentrations urinaires de BPA et des mesures de l'IMC et du tour de taille.

Résultats : On a pu associer positivement la concentration urinaire de BPA et l'obésité (définie par l'IMC). Le rapport de cotes s'est situé à 1,54 (intervalle de confiance [IC] à $95 \%: 1,002$ à 2,37) dans le quartile de BPA le plus élevé par rapport au plus bas (test de tendance, $p=0,041$ ). La concentration urinaire de BPA n'a pas été associée au tour de taille pour les valeurs seuils courantes. Enfin, chaque unité d'augmentation du log naturel de la concentration urinaire de BPA a été associée à une hausse de $0,33 \mathrm{~kg} / \mathrm{m}^{2}$ (IC à $95 \%: 0,10$ à 0,57 ) de l'IMC et de $1,00 \mathrm{~cm}$ (IC à $95 \%: 0,34$ à 1,65) du tour de taille.

Conclusion : Notre étude vient enrichir le corpus croissant de données probantes sur l'association positive entre BPA et obésité. Des études prospectives comportant un suivi de mesures sont nécessaires pour définir la dimension temporelle de l'association et améliorer la classification de l'exposition.

Mots-clés : bisphénol A, perturbateurs endocriniens, obésité, indice de masse corporelle, tour de taille, biosurveillance, Enquête canadienne sur les mesures de la santé

\section{Introduction}

L’obésité est une épidémie mondiale en augmentation qui devrait dépasser le tabagisme pour se situer au premier rang des facteurs contribuant au fardeau des maladies chroniques ${ }^{1,2}$. Au Canada, un adulte sur quatre est obèse ${ }^{3}$. La consommation excessive d'aliments riches en calories, une activité physique insuffisante et une
Points saillants

- Cette étude est la première au Canada à se pencher sur l'association entre le bisphénol A (BPA) et les mesures de l'adiposité.

- Plus la concentration urinaire de BPA est élevée, plus le risque d'obésité est grand chez les adultes canadiens de 18 à 79 ans.

- La concentration urinaire de BPA est associée positivement à l'adiposité, soit l'indice de masse corporelle et le tour de taille.

- Des études prospectives comportant un suivi des mesures sont nécessaires pour définir la dimension temporelle de l'association et améliorer la classification de l'exposition.

augmentation de la sédentarité sont considérées comme les principaux facteurs responsables de cette épidémie ${ }^{4}$.

Compte tenu de l'augmentation importante de l'obésité au cours des dernières décennies, on a exploré diverses hypothèses sur l'existence d'autres facteurs de risque potentiellement modifiables qui s'ajouteraient au déséquilibre énergétique. Des études écologiques ont fait ressortir une corrélation entre la prévalence croissante de l'obésité et la hausse de la production de produits chimiques synthétiques, dont le bisphénol A (BPA) ${ }^{5}$. Le BPA est un perturbateur endocrinien répandu dans les aliments et les boissons conservés dans des contenants en plastique polycarbonate et en résines époxydes ${ }^{6}$. La principale voie d'exposition au BPA est la nourriture, mais l'exposition par voie cutanée est aussi

Rattachement des auteurs :

1. Agence de la santé publique du Canada, Ottawa (Ontario), Canada

2. École de santé publique Dalla Lana, Université de Toronto, Toronto (Ontario), Canada

3. Département des sciences de la santé, Université Carleton, Ottawa (Ontario), Canada

4. École Gillings de santé publique mondiale, Université de Caroline du Nord, Chapel Hill (Caroline du Nord), États-Unis

Correspondance : Minh T. Do, Division de la surveillance et de l'épidémiologie, Agence de la santé publique du Canada, 785, avenue Carling, Ottawa (Ontario) K1A 0K9; tél. : 613-797-7587; téléc. : 613-941-2057; courriel : minht.do@canada.ca 
possible, par contact de la peau avec du papier thermique (p. ex. reçus, billets) ${ }^{6}$. Plus de $90 \%$ des Canadiens ${ }^{7}$ et des Américains $^{8}$ présentent des quantités détectables de BPA dans leur urine, signe d'une large exposition chez l'humain.

Des études in vitro ont montré que le BPA augmente la différenciation des adipocytes, entraînant l'accumulation excessive de tissus adipeux ${ }^{9}$. Des études menées sur des rongeurs ont aussi révélé que l'exposition au BPA accroît la masse adipeuse et favorise la prise de poids ${ }^{10}$. Plus récemment, des données probantes ont relevé l'existence d'une relation entre le BPA et l'obésité chez l'humain, mais elles se limitaient à un faible effectif de population. Des études épidémiologiques réalisées aux États-Unis ${ }^{11}$, en Chine ${ }^{13}$ et en Corée ${ }^{14,15}$ ont fait ressortir des associations positives entre le BPA et des mesures de l'adiposité chez les adultes. De plus, le BPA est un composé œstrogénique dont l'effet varie en fonction du sexe dans les modèles animaux $^{16}$, les données indiquant des différences en fonction du sexe dans l'association entre le BPA et l'obésité demeurant cependant limitées chez l'humain.

L'Enquête canadienne sur les mesures de la santé (ECMS) est une enquête qui collecte depuis 2007 des données de biosurveillance et des données anthropométriques auprès d'un échantillon national représentatif de la population canadienne. Étant donné que le BPA est répandu au Canada et que les données appuient l'existence d'un lien entre le BPA et l'obésité, nous avons étudié, à la fois globalement et par sexe, les associations entre les concentrations urinaires de BPA et les mesures de l'adiposité chez les adultes de 18 à 79 ans, à l'aide des données de l'ECMS.

\section{Méthodologie}

\section{Source de données et population à l'étude}

Cette étude repose sur les données des cycles 1 (2007-2009) et 2 (2009-2011) de l'ECMS, qui est décrite en détail ailleurs ${ }^{17,18}$. Pour résumer, il s'agit d'une enquête transversale continue fondée sur un plan d'échantillonnage stratifié à plusieurs degrés qui vise à collecter des données représentatives de l'ensemble des ménages canadiens, exclusion faite des personnes vivant dans des réserves et d'autres établissements autochtones, des membres à temps plein des Forces canadiennes et des résidents d'établissements et de certaines régions éloignées. Les données ont été recueillies auprès de 5604 personnes de 6 à 79 ans dans 15 sites répartis au Canada pour le cycle 1 et auprès de 6395 personnes âgées de 3 à 79 ans dans 18 sites pour le cycle 2. L'échantillon représentait pour chaque cycle environ $96 \%$ de la population canadienne du groupe d’âge ciblé.

L'ECMS repose sur une entrevue à domicile portant sur des caractéristiques sociodémographiques, le mode de vie et la santé, suivie d'une visite dans un centre d'examen mobile (CEM) visant à prendre des mesures physiques directes, notamment des mesures anthropométriques et des prélèvements de sang et d'urine. Le consentement éclairé de chaque participant a été obtenu par écrit. Les protocoles ont été vérifiés et approuvés par le comité d'éthique de la recherche de Santé Canada et de l'Agence de la santé publique du Canada (ASPC).

Les analyses réalisées dans cette étude ont été limitées aux adultes de 18 à 79 ans au moment de la visite au CEM (cycle 1 : $\mathrm{n}=3$ 726; cycle $2: \mathrm{n}=3$ 873). Nous avons regroupé les données des deux cycles de l'ECMS pour accroître la taille de l'échantillon et l'exactitude statistique des estimations $^{19}$. Bien que tous les participants ayant fourni des échantillons d'urine lors du cycle 1 ( $n=3$ 702) aient été admissibles à des mesures du BPA, seul un sousgroupe aléatoire de participants du cycle 2 ( $n=1$ 117) a été retenu pour ces mesures, ce qui a donné un échantillon combiné de 4819 participants. Après exclusion des femmes enceintes $(n=37)$ et des participants dont la concentration urinaire de BPA ou de créatinine était manquante ( $n=49$ ), notre échantillon final était de 4733 participants.

\section{Mesures en laboratoire}

Des échantillons ponctuels d'urine ont été recueillis : urines de milieu de jet lors du cycle 1 et de premier jet lors du cycle 2 . Les participants du cycle 2 devaient également s'abstenir d'uriner durant les deux heures précédant leur visite au CEM. Le protocole a été modifié afin de permettre l'utilisation des nouveaux tests de détection des marqueurs de maladies infectieuses dans le cycle $2^{18}$. Malgré ces changements, les concentrations urinaires de BPA sont demeurées semblables d'un cycle à l'autre ${ }^{6}$. Après la collecte et l'aliquotage, les échantillons d'urine ont été congelés à $-20{ }^{\circ} \mathrm{C}$ et expédiés sur de la glace sèche au laboratoire d'analyse de l'Institut national de santé publique du Québec (INSPQ) à Québec (Québec, Canada).

Les concentrations de BPA total (formes libre et conjuguée) ont été mesurées par une chromatographie en phase gazeuse couplée à une spectrophotométrie de masse en tandem, conformément à des méthodes décrites antérieurement ${ }^{7,20}$. La limite de détection (LD) était de 0,2 $\mu \mathrm{g} / \mathrm{L}$ dans les deux cycles. Nous avons attribué aux concentrations inférieures à la $\mathrm{LD}$ ( $\mathrm{n}=436 ; 9 \%$ ) la valeur de $\mathrm{LD} / 2(0,1 \mu \mathrm{g} / \mathrm{L})$. Les concentrations urinaires de BPA ont été réparties en quartiles (moins de $0,7 \mu \mathrm{g} / \mathrm{L}$, 0,7 à $1,2 \mu \mathrm{g} / \mathrm{L}, 1,3$ à $2,4 \mu \mathrm{g} / \mathrm{L}$ et plus de $2,4 \mu \mathrm{g} / \mathrm{L}$ ) en fonction de la distribution dans la population à l'étude. Par ailleurs, les concentrations de BPA ont été transformées en log naturel en raison de la distribution asymétrique des valeurs et ont été analysées comme une variable continue.

Pour tenir compte de la dilution de l'urine, on a utilisé la créatinurie, que l'on a mesurée à l'aide de la méthode colorimétrique de Jaffée ${ }^{21}$. Les concentrations inférieures à la LD (cycle 1 : $0,035 \mathrm{~g} / \mathrm{L}$; cycle 2 : 0,050 g/L) ont été codées comme " manquantes » et exclues des analyses $(n=9)$, conformément aux instructions de Statistique Canada ${ }^{19}$. Pour réduire le biais potentiel associé aux différences systématiques sur le plan de la créatinurie en fonction des caractéristiques de la population ( $p$. ex. sexe, âge, origine ethnique), nous avons inclus la créatinine comme covariable dans tous les modèles plutôt que de normaliser les concentrations de BPA en fonction de la créatinine ${ }^{22}$.

\section{Mesures anthropométriques}

Notre principal résultat d'intérêt était l'indice de masse corporelle (IMC), calculé d'après la taille et le poids selon des procédures normalisées ${ }^{23}$. La taille a été mesurée à $0,01 \mathrm{~cm}$ près au moyen d'un stadiomètre numérique ProScale M150 (Accurate Technology Inc., Fletcher, Caroline du Nord, États-Unis) et le poids à $0,1 \mathrm{~kg}$ près au moyen d'un pèse-personne Mettler Toledo VLC avec terminal Panther Plus (Mettler Toledo Canada, Mississauga, Ontario, Canada). L'IMC a été calculé en divisant le poids $(\mathrm{kg})$ par le carré de la taille $\left(\mathrm{m}^{2}\right)$ puis réparti en catégories : poids 
insuffisant (moins de $18,5 \mathrm{~kg} / \mathrm{m}^{2}$ ), poids normal $\left(18,5\right.$ à $\left.24,9 \mathrm{~kg} / \mathrm{m}^{2}\right)$, embonpoint $\left(25,0\right.$ à $\left.29,9 \mathrm{~kg} / \mathrm{m}^{2}\right)$, obésité de classe I $\left(30,0\right.$ à $\left.34,9 \mathrm{~kg} / \mathrm{m}^{2}\right)$ et obésité de classe II ou III $\left(35,0 \mathrm{~kg} / \mathrm{m}^{2} \text { et plus }\right)^{4}$.

Nous avons aussi tenu compte du tour de taille, qui constitue un indicateur de la graisse abdominale et qui a été mesuré à $0,1 \mathrm{~cm}$ près à la fin d'une expiration normale à mi-chemin entre la dernière côte flottante et le haut de la crête iliaque ${ }^{4}$. L'obésité abdominale a été définie sur la base de valeurs seuils du tour de taille en fonction du sexe : $102 \mathrm{~cm}$ et plus chez les hommes et $88 \mathrm{~cm}$ et plus chez les femmes ${ }^{4,24}$.

\section{Facteurs potentiels de confusion}

Nous avons défini les facteurs de confusion potentiels à partir de la littérature sur l'association entre le BPA et l'obésité chez les adultes ${ }^{11-15}$. Nous avons choisi comme variables sociodémographiques le sexe, l'âge (18 à 29 ans, 30 à 39 ans, 40 à 49 ans, 50 à 59 ans, 60 à 69 ans et 70 ans et plus), l'origine ethnique (Blanc, non-Blanc), le niveau de scolarité (études secondaires non terminées, diplôme d'études secondaires, diplôme d'études postsecondaires) et la suffisance de revenu du ménage (classe inférieure ou classe moyenne inférieure, classe moyenne supérieure, classe supérieure) évaluée à partir du revenu annuel du ménage et du nombre de membres du ménage. Nous avons aussi pris en compte des facteurs liés au mode de vie : le tabagisme (personnes n'ayant jamais fumé, ex-fumeurs, fumeurs), la consommation d'alcool (0 à 3 fois par mois, 1 à 6 fois par semaine, tous les jours) et l'activité physique, évaluée à partir de la dépense énergétique quotidienne moyenne pendant les activités de loisir des 3 derniers mois. Les participants ont été classés comme actifs (3,0 $\mathrm{kcal} / \mathrm{kg} / \mathrm{j}$ et plus), modérément actifs (1,5 à 2,9 kcal/kg/j) ou inactifs (moins de $1,5 \mathrm{kcal} / \mathrm{kg} / \mathrm{j})$.

Comme la nourriture est à la fois la principale source d'exposition au BPA et un facteur de risque d'obésité connu, nous avons pris en compte les facteurs potentiels de confusion associés en établissant plusieurs mesures alimentaires à partir du questionnaire semi-quantitatif sur la fréquence de consommation des aliments de l'ECMS, dans lequel les participants devaient indiquer le nombre de fois (par jour, par semaine, par mois ou par année) où ils avaient consommé certains types d'aliments ou de boissons au cours de la dernière année. Un score de qualité de l'alimentation a été calculé à l'aide d'une méthode similaire à celle du score de régime méditerranéen ${ }^{25}$. Dans un premier temps, nous avons classé les produits alimentaires en sept catégories (fruits; légumes; légumineuses et noix; céréales et grains; lait et produits laitiers; poisson et fruits de mer; viande rouge ou transformée) puis nous avons calculé les apports médians (nombre de fois par jour) pour chacun d'eux, selon le sexe. Toutes les catégories, sauf celle de la viande rouge ou transformée, ont été considérées comme bénéfiques, de sorte que la valeur de « 1 » a été attribuée aux participants dont l'apport était supérieur à la médiane (et inversement, à ceux dont l'apport en viande rouge ou transformée était inférieur à la médiane). Le score global de qualité de l'alimentation a été établi en additionnant les valeurs associées à chaque catégorie : un score de 0 à 3 a été considéré comme faible, un score de 4 ou 5, comme moyen, et un score de 6 ou 7, comme élevé. Nous avons aussi examiné la fréquence de consommation des aliments et boissons pouvant contenir du BPA et de ceux connus pour favoriser l'obésité en raison de leur haute teneur énergétique, comme les boissons sucrées (boissons gazeuses régulières, boissons pour sportifs, boissons à saveur de fruits, etc.), qui ont été réparties en trois catégories (moins d'une fois par semaine, 1 à 6 fois par semaine et 1 fois ou plus par jour), et la malbouffe (pommes de terre frites, croustilles régulières, tortillas et croustilles de maïs, crème glacée, etc.), répartie également en trois catégories (moins d'une fois par semaine, 1 à 4 fois par semaine et 5 fois par semaine ou plus).

\section{Analyses statistiques}

Pour tenir compte du plan d'échantillonnage complexe de l'ECMS, nous avons utilisé des poids d'échantillonnage dans toutes les analyses ${ }^{17,18}$. Nous avons obtenu des estimations de la variance à l'aide de la méthode d'auto-amorçage (bootstrap). Nous avons réalisé nos analyses à l'aide de la version $9.3 \mathrm{du}$ logiciel SAS (SAS Institute Inc., Cary, Caroline du Nord, États-Unis) et de la version 11.0.1 du logiciel SUDAAN (Research Triangle Institute, Research Triangle Park, Caroline du Nord, ÉtatsUnis). Nous avons établi le seuil de signification statistique à $p<0,05$, et les analyses étaient bilatérales.
Nous avons effectué des analyses descriptives pour rendre compte de la distribution des caractéristiques dans l'ensemble de la population et par quartile de BPA. Nous avons utilisé le test du khi carré de RaoScott pour déterminer si la distribution des quartiles de BPA variait en fonction des catégories de chaque caractéristique. Nous avons aussi calculé la moyenne géométrique (MG) des concentrations urinaires de BPA et nous avons évalué les différences à l'aide du test $t$ avec des valeurs $p$ corrigées selon la méthode de Bonferroni, en fonction du nombre de comparaisons.

Nous avons utilisé une régression logistique multinomiale pour examiner les associations entre les quartiles de BPA urinaire et l'IMC, catégorisé en poids insuffisant ou normal (moins de $25,0 \mathrm{~kg} / \mathrm{m}^{2}$; catégorie de référence), embonpoint (25,0 à 29,9 kg/ $\left.\mathrm{m}^{2}\right)$ ou obésité $\left(30,0 \mathrm{~kg} / \mathrm{m}^{2}\right.$ et plus). Nous avons effectué des tests de tendance pour l'ensemble des quartiles par modélisation de la médiane de chaque quartile de BPA considérée comme une variable continue. Nous avons aussi considéré comme une variable continue, dans un autre modèle, la concentration de BPA transformée en log naturel. Pour évaluer l'association entre la concentration urinaire de BPA et l'obésité abdominale (c.-à-d. un tour de taille élevé), nous avons appliqué une régression logistique binaire. Nous avons ajusté tous les modèles de base en fonction du sexe, de l'âge (en années, variable continue) et de la créatinurie (g/L, variable continue). Nous avons ensuite établi des modèles multidimensionnels en ajoutant aux modèles de base des variables individuelles provenant de la liste des facteurs potentiels de confusion. Nous avons inclus les variables dans les modèles finaux si elles présentaient une association avec à la fois l'exposition (quartile de BPA; $p<0,20$ d'après le test du khi carré) et le résultat (catégorie d'IMC ou de tour de taille; prise en compte dans le modèle de régression si $p<0,20)$ ou si elles modifiaient de plus de $10 \%$ le rapport de cotes ajusté en fonction du sexe, de l'âge ou de la créatinurie. Outre le sexe, l'âge et la créatinurie, nous avons ajusté tous les modèles d'IMC et de tour de taille en fonction de l'origine ethnique, de la consommation d'alcool et de la consommation de malbouffe, ainsi que les modèles de tour de taille en fonction de la consommation de boissons sucrées. Nous avons de plus estimé les associations entre la concentration urinaire de BPA (quartiles ou variable continue transformée en log naturel) à l'aide de mesures continues 
de l'IMC et du tour de taille faisant appel à une régression linéaire. Enfin, nous avons stratifié nos analyses pour explorer si l'effet potentiel variait avec le sexe. Nous avons aussi vérifié la signification statistique des interactions multiplicatives (sexe $\times$ BPA).

Nous avons mené diverses analyses de sensibilité. Premièrement, comme l'état de jeûne (10 heures et plus ou moins de 10 heures) et le moment de la collecte d'urine (matin, après-midi ou soir) sont susceptibles d'influencer les concentrations urinaires de $\mathrm{BPA}^{26,27}$, nous avons évalué leur effet de confusion potentiel. Deuxièmement, nous avons limité nos modèles aux participants de 18 à 64 ans, car les classifications courantes de l'IMC et du tour de taille ne sont pas systématiquement applicables aux personnes âgées ${ }^{24}$. Troisièmement, nous avons reconduit nos analyses par modèles en excluant les participants ayant déclaré une affection chronique (maladie cardiovasculaire [crise cardiaque, accident vasculaire cérébral, toute cardiopathie], diabète ou maladie rénale) diagnostiquée par un professionnel de la santé et susceptible d'être liée à l'obésité, à l'exposition au BPA ou à l'excrétion de $\mathrm{BPA}^{28,29}$. Enfin, comme la distribution des quartiles de BPA urinaire variait au sein de notre échantillon d'ensemble en fonction du sexe, nous avons reconduit nos analyses par modèles stratifiés selon le sexe en utilisant les quartiles spécifiques à chaque sexe au lieu des quartiles d'ensemble.

\section{Résultats}

Les caractéristiques de la population à l'étude et les concentrations urinaires de BPA sont présentées dans le tableau 1. La MG des concentrations urinaires de BPA était de $1,17 \mu \mathrm{g} / \mathrm{L}$ (IC à $95 \%: 1,10$ à 1,24 ) pour l'ensemble. Elle diminuait en fonction de l'âge et s'est révélée significativement plus élevée chez les hommes et chez les participants qui consommaient de la malbouffe 5 fois par semaine ou plus ( $p<0,05$ dans tous les cas; données non présentées). Hormis le sexe ( $p=0,023)$, aucune des caractéristiques n’a été associée à une modification de la distribution des quartiles de BPA.

Comme l'indique le tableau 1, $60 \%$ des adultes canadiens faisaient de l'embonpoint (35\%) ou étaient obèses (25\%) et $35 \%$ présentaient un tour de taille élevé (c.-à-d. une obésité abdominale). La MG des concentrations urinaires de BPA augmentait en fonction de l'IMC, de respectivement $0,84 \mu \mathrm{g} / \mathrm{L}$ (IC à $95 \%: 0,42$ à 1,67 ) chez les personnes ayant un poids insuffisant et $1,09 \mu \mathrm{g} / \mathrm{L}$ (IC à $95 \%$ : 0,96 à 1,23) chez celles ayant un poids normal à $1,34 \mu \mathrm{g} / \mathrm{L}$ (IC à $95 \%$ : 1,13 à 1,59) chez celles classées dans les catégories d'obésité II ou III (valeur $p$ pour la tendance $=0,06$ ). Les concentrations de BPA ne variaient pas en fonction des catégories de tour de taille.

Le tableau 2 présente les associations entre, d'une part, la concentration urinaire de BPA et, d'autre part, l'embonpoint et l'obésité au sens de l'IMC, et ce, dans l'échantillon total et selon le sexe. Dans le modèle global ajusté en fonction du sexe, de l'âge et de la créatinurie (modèle 1), la probabilité d'être obèse (par rapport à celle d'avoir un poids insuffisant ou normal) était significativement plus élevée chez les participants du quartile de BPA le plus élevé que chez ceux du quartile le moins élevé (RC $=1,56$, IC à $95 \%: 1,02$ à 2,38). Cette tendance augmentait d'un quartile à l'autre (valeur $p$ pour la tendance $=0,036$ ). Les résultats sont demeurés largement inchangés après ajustement supplémentaire

TABLEAU 1

Moyenne géométrique et distribution des concentrations urinaires de bisphénol A selon les caractéristiques individuelles des adultes canadiens de 18 à 79 ans ( $n=4$ 733), Enquête canadienne sur les mesures de la santé, 2007-2011

\begin{tabular}{|c|c|c|c|c|c|c|c|c|c|}
\hline \multirow{2}{*}{ Caractéristiques } & \multirow[t]{2}{*}{$\mathbf{N}^{\mathbf{a}}$} & \multirow[t]{2}{*}{$\%^{\mathbf{b}}$} & \multirow{2}{*}{$\underset{(\mu \mathrm{g} / \mathrm{L})}{\text { MG }}$} & \multirow{2}{*}{ IC à $95 \%$} & \multicolumn{4}{|c|}{$\begin{array}{l}\text { Quartile de BPA urinaire } \\
(\text { en } \%)^{b, c}\end{array}$} & \multirow[t]{2}{*}{ Valeur $p^{d}$} \\
\hline & & & & & 1 & 2 & 3 & 4 & \\
\hline Total & 4733 & 100 & 1,17 & 1,10 à 1,24 & 26,3 & 23,3 & 25,3 & 25,1 & \\
\hline Sexe & & & & & & & & & 0,023 \\
\hline Hommes & 2275 & 49,8 & 1,27 & 1,17 à 1,38 & 22,7 & 23,3 & 27,3 & 26,8 & \\
\hline Femmes & 2458 & 50,2 & 1,08 & 0,99 à 1,17 & 29,9 & 23,4 & 23,3 & 23,5 & \\
\hline Âge (ans) & & & & & & & & & 0,076 \\
\hline 18 à 29 & 894 & 21,6 & 1,35 & 1,11 à 1,65 & $22,2^{\mathrm{E}}$ & 24,3 & 25,2 & 28,3 & \\
\hline 30 à 39 & 899 & 17,4 & 1,34 & 1,19 à 1,50 & 22,5 & 22,1 & 27,4 & 28,1 & \\
\hline 40 à 49 & 967 & 21,1 & 1,07 & 0,91 à 1,27 & 28,8 & 21,3 & 22,2 & 27,7 & \\
\hline 50 à 59 & 602 & 18,8 & 1,18 & 0,98 à 1,44 & $23,6^{\mathrm{E}}$ & 25,0 & 31,1 & 20,3 & \\
\hline 60 à 69 & 858 & 13,8 & 0,99 & 0,83 à 1,17 & 30,8 & 26,5 & 22,7 & 20,0 & \\
\hline 70 et plus & 513 & 7,4 & 0,94 & 0,78 à 1,14 & 38,2 & 19,2 & 19,4 & 23,2 & \\
\hline Origine ethnique & & & & & & & & & 0,096 \\
\hline Blancs & 3981 & 81,4 & 1,18 & 1,10 à 1,26 & 26,1 & 24,7 & 24,2 & 25,0 & \\
\hline Non-Blancs & 747 & $18,6^{\mathrm{E}}$ & 1,14 & 0,93 à 1,39 & 27,0 & 17,2 & 30,0 & 25,7 & \\
\hline Niveau de scolarité & & & & & & & & & 0,681 \\
\hline Études secondaires non terminées & 656 & 12,2 & 1,11 & 0,94 à 1,32 & 29,0 & 26,5 & 21,2 & 23,2 & \\
\hline Diplôme d'études secondaires & 1271 & 28,9 & 1,14 & 0,99 à 1,32 & 25,3 & 25,7 & 25,4 & 23,6 & \\
\hline Diplôme d'études postsecondaires & 2758 & 59,0 & 1,19 & 1,10 à 1,29 & 26,2 & 21,9 & 25,6 & 26,4 & \\
\hline
\end{tabular}


TABLEAU 1 (suite)

Moyenne géométrique et distribution des concentrations urinaires de bisphénol A selon les caractéristiques individuelles des adultes canadiens de 18 à 79 ans $(n=4$ 733), Enquête canadienne sur les mesures de la santé, 2007-2011

\begin{tabular}{|c|c|c|c|c|c|c|c|c|c|}
\hline Caractéristiques & $\mathbf{N}^{\mathbf{a}}$ & $\%^{\mathrm{b}}$ & $\begin{array}{c}\text { MG } \\
(\mu \mathrm{g} / \mathrm{L})\end{array}$ & IC à $95 \%$ & \multicolumn{4}{|c|}{$\begin{array}{l}\text { Quartile de BPA urinaire } \\
(\text { (en \% })^{\text {b,c }}\end{array}$} & Valeur $p$ \\
\hline Suffisance du revenu du ménage & & & & & & & & & 0,534 \\
\hline Classe inférieure ou moyenne inférieure & 1063 & 19,2 & 1,29 & 1,17 à 1,43 & 23,9 & 21,1 & 29,1 & 26,0 & \\
\hline Classe moyenne supérieure & 1586 & 31,0 & 1,09 & 0,97 à 1,23 & 29,0 & 23,0 & 25,1 & 23,0 & \\
\hline Classe supérieure & 2084 & 49,8 & 1,18 & 1,08 à 1,27 & 25,5 & 24,5 & 23,9 & 26,1 & \\
\hline Jamais fumé & 2365 & 51,6 & 1,11 & 1,02 à 1,21 & 27,9 & 22,4 & 24,7 & 25,0 & \\
\hline Ex-fumeur & 1416 & 27,1 & 1,15 & 1,05 à 1,27 & 26,6 & 24,1 & 26,6 & 22,7 & \\
\hline Fumeur & 952 & 21,3 & 1,34 & 1,14 à 1,58 & 22,0 & 24,7 & 24,9 & 28,5 & \\
\hline Consommation d'alcool & & & & & & & & & 0,126 \\
\hline 0 à 3 fois/mois & 2426 & 50,3 & 1,16 & 1,07 à 1,26 & 27,5 & 21,6 & 24,6 & 26,3 & \\
\hline Actif & 1037 & 21,1 & 1,27 & 1,12 à 1,45 & 24,0 & 20,7 & 26,4 & 28,9 & \\
\hline Modérément actif & 1215 & 24,5 & 1,17 & 1,02 à 1,35 & 26,4 & 23,0 & 26,0 & 24,6 & \\
\hline Inactif & 2481 & 54,5 & 1,13 & 1,04 à 1,22 & 27,1 & 24,5 & 24,5 & 23,9 & \\
\hline Score de qualité de l'alimentation & & & & & & & & & 0,515 \\
\hline Faible (0 à 3) & 1998 & 43,7 & 1,26 & 1,14 à 1,39 & 25,0 & 21,5 & 26,4 & 27,1 & \\
\hline Moyen (4 à 5) & 1922 & 40,1 & 1,14 & 1,01 à 1,27 & 25,7 & 24,5 & 26,2 & 23,6 & \\
\hline Élevé (6 à 7) & 781 & 16,2 & 1,05 & 0,88 à 1,24 & 30,8 & 24,8 & $20,8^{\mathrm{E}}$ & 23,6 & \\
\hline Consommation de boissons sucrées & & & & & & & & & 0,182 \\
\hline Moins d'une fois/semaine & 2565 & 53,2 & 1,09 & 1,00 à 1,20 & 28,5 & 24,1 & 24,8 & 22,6 & \\
\hline 1 à 6 fois/semaine & 1419 & 31,1 & 1,26 & 1,16 à 1,38 & 22,9 & 23,3 & 26,0 & 27,8 & \\
\hline Poids insuffisant (moins de 18,5 ) & 69 & $1,9^{\mathrm{E}}$ & 0,84 & 0,42 à 1,67 & - $^{\mathrm{F}}$ & $49,0^{\mathrm{E}}$ & - $^{\mathrm{F}}$ & $-^{\mathrm{F}}$ & \\
\hline Poids normal $(18,5$ à 24,9$)$ & 1730 & 37,5 & 1,09 & 0,96 à 1,23 & 28,6 & 24,0 & 24,9 & 22,6 & \\
\hline Embonpoint $(25,0$ à 29,9$)$ & 1737 & 35,2 & 1,21 & 1,08 à 1,36 & 24,9 & 22,2 & 26,7 & 26,2 & \\
\hline Obésité de classe I $(30,0$ à 34,9) & 744 & 16,1 & 1,23 & 1,00 à 1,51 & 28,2 & 21,6 & 20,5 & 29,7 & \\
\hline Obésité de classe II ou III (35,0 et plus) & 442 & 9,3 & 1,34 & 1,13 à 1,59 & 18,8 & $22,8^{\mathrm{E}}$ & 33,0 & $25,4^{\mathrm{E}}$ & \\
\hline Tour de taille élevé & & & & & & & & & 0,823 \\
\hline Non & 2931 & 64,7 & 1,17 & 1,08 à 1,27 & 26,0 & 22,6 & 26,4 & 25,0 & \\
\hline Oui (hommes $\geq 102 \mathrm{~cm}$; femmes $\geq 88 \mathrm{~cm}$ ) & 1781 & 35,3 & 1,17 & 1,04 à 1,33 & 27,0 & 23,7 & 23,6 & 25,7 & \\
\hline
\end{tabular}

Abréviations : BPA, bisphénol A; IC, intervalle de confiance; MG, moyenne géométrique.

Remarque : Les pourcentages et les MG ont été pondérés à l'aide de poids d'échantillonnage.

${ }^{a}$ Il est possible que la somme des effectifs ne corresponde pas à la population totale $(n=4733)$ en raison de données manquantes pour certaines variables.

${ }^{\mathrm{b}}$ Il est possible que la somme des pourcentages ne soit pas de $100 \%$ en raison de l'arrondissement des valeurs.

${ }^{c}$ Quartile $1:$ moins de $0,7 \mu \mathrm{g} / \mathrm{L}$; quartile $2: 0,7$ à $1,2 \mu \mathrm{g} / \mathrm{L}$; quartile $3: 1,3$ à 2,4 $\mu \mathrm{g} / \mathrm{L}$; quartile $4:$ plus de $2,4 \mu \mathrm{g} / \mathrm{L}$.

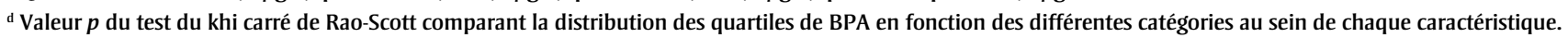

E À interpréter avec prudence (le coefficient de variation se situe entre $16,6 \%$ et $33,3 \%$ ).

F Les données ne satisfont pas aux lignes directrices de Santé Canada pour la diffusion en raison de leur variabilité extrême (coefficient de variation supérieur à 33,3 \%). 
TABLEAU 2

Associations entre la concentration urinaire de bisphénol $\mathrm{A}$ et l'embonpoint et l'obésité (par rapport à un poids insuffisant ou normal) chez les adultes de 18 à 79 ans, globalement et par sexe, Enquête canadienne sur les mesures de la santé, 2007-2011

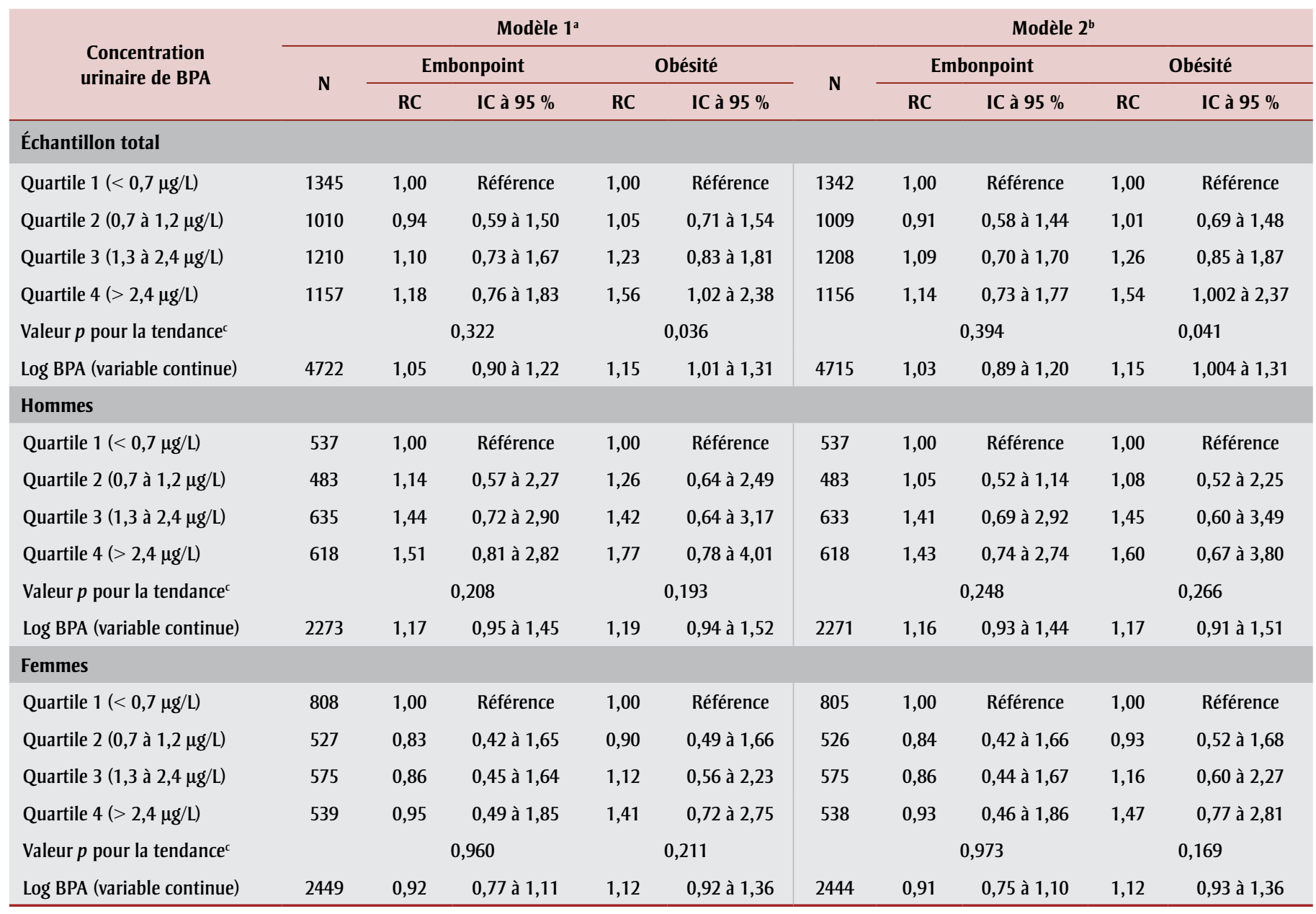

Abréviations : BPA, bisphénol A; IC, intervalle de confiance; RC, rapport de cotes.

${ }^{a}$ Ajusté en fonction du sexe (modèle global seulement), de l'âge et de la créatinurie.

${ }^{\mathrm{b}}$ Ajusté en fonction des covariables du modèle 1 ainsi que de l'origine ethnique, de la consommation d'alcool et de la consommation de malbouffe.

c Valeur p du test de tendance, calculée par modélisation de la médiane de chaque quartile de BPA comme une variable continue.

en fonction des facteurs potentiels de confusion (modèle 2) (RC [quartile 4 par rapport à quartile 1] $=1,54$, IC à $95 \%: 1,002$ à 2,37 ; valeur $p$ pour la tendance $=0,041$ ) De même, le BPA transformé en log naturel (valeur continue) a été positivement associé à l'obésité $(\mathrm{RC}=1,15$, IC à $95 \%$ : 1,004 à 1,31). Les associations étaient généralement positives mais non significatives dans la catégorie de l'embonpoint, avec un RC à 1,14 (IC à $95 \%: 0,73$ à 1,77 ) dans le quartile de BPA le plus élevé (par rapport au quartile le moins élevé). Lors de la ventilation par sexe, les associations entre la concentration urinaire de BPA et tant l'embonpoint que l'obésité ne se sont révélées statistiquement significatives pour aucun des deux sexes. Bien que l'existence d'une interaction significative entre le sexe et le BPA n'ait pas pu été démontrée ( $p>0,05$; données non présentées), nous avons observé des associations positives plus marquées chez les hommes que chez les femmes aussi bien avec l'embonpoint (RC [quartile 4 par rapport au quartile 1] = 1,43 contre 0,93) qu'avec l'obésité (RC [quartile 4 par rapport au quartile 1$]=1,60$ contre 1,47$)$.

Nous n'avons observé aucune association significative entre le BPA urinaire et l'obésité abdominale, et ce, ni pour les participants dans leur ensemble (RC [quartile 4 par rapport au quartile 1] $=1,16$, IC à $95 \%: 0,81$ à 1,66 ; valeur $p$ pour la tendance $=0,463$ ) ni pour l'un ou l'autre des sexes (tableau 3). Les modèles stratifiés selon le sexe indiquaient bien une association plus forte chez les hommes que chez les femmes (RC [quartile 4 par rapport au quartile 1] = 1,28 contre 1,03$)$, mais aucune interaction statistiquement significative n'a pu être relevée ( $p>0,05$; données non présentées). L'ajustement supplémentaire des modèles en fonction de la taille, destinée à tenir compte de la stature générale, n’a eu aucune incidence sur les résultats (données non présentées).

La figure 1 présente les associations entre les divers quartiles de BPA urinaire et les mesures de l'IMC et du tour de taille. Les participants des quartiles 3 et 4 avaient un IMC significativement plus élevé $(1,03 \mathrm{~kg} /$ $\mathrm{m}^{2}$, IC à $95 \%: 0,30$ à 1,76$)$ que ceux du quartile $1\left(1,06 \mathrm{~kg} / \mathrm{m}^{2}\right.$, IC à $95 \%$ : 0,18 à 1,93). Une tendance semblable a été observée pour le tour de taille, mais la signification statistique a été atteinte uniquement pour le quartile $3(2,42 \mathrm{~cm}$, IC à $95 \%: 0,46$ à 4,39 ) et non pour le 
TABLEAU 3

Associations entre la concentration urinaire de bisphénol $A$ et l'obésité abdominale $^{a}$ chez les adultes de 18 à 79 ans, globalement et par sexe, Enquête canadienne sur les mesures de la santé, 2007-2011

\begin{tabular}{|c|c|c|c|c|c|c|}
\hline \multirow{2}{*}{$\begin{array}{c}\text { Concentration urinaire } \\
\text { de BPA }\end{array}$} & \multicolumn{3}{|c|}{ Modèle $1^{\text {b }}$} & \multicolumn{3}{|c|}{ Modèle $2^{c}$} \\
\hline & $\mathbf{N}$ & RC & IC à $95 \%$ & $\mathbf{N}$ & RC & IC à $95 \%$ \\
\hline \multicolumn{7}{|l|}{ Échantillon total } \\
\hline Quartile 1 (< 0,7 $\mu \mathrm{g} / \mathrm{L})$ & 1342 & 1,00 & Référence & 1339 & 1,00 & Référence \\
\hline Quartile $2(0,7$ à $1,2 \mu \mathrm{g} / \mathrm{L})$ & 1003 & 1,12 & 0,76 à 1,67 & 1001 & 1,12 & 0,75 à 1,65 \\
\hline Quartile 3 (1,3 à 2,4 $\mu \mathrm{g} / \mathrm{L})$ & 1211 & 0,96 & 0,64 à 1,45 & 1209 & 0,99 & 0,67 à 1,46 \\
\hline Quartile 4 (> 2,4 $\mu \mathrm{g} / \mathrm{L})$ & 1156 & 1,16 & 0,81 à 1,65 & 1155 & 1,16 & 0,81 à 1,66 \\
\hline Valeur $p$ pour la tendance ${ }^{d}$ & \multicolumn{4}{|c|}{0,470} & \multicolumn{2}{|c|}{0,463} \\
\hline Log BPA (variable continue) & 4712 & 1,06 & 0,96 à 1,16 & 4704 & 1,05 & 0,96 à 1,16 \\
\hline \multicolumn{7}{|l|}{ Hommes } \\
\hline Quartile $1(<0,7 \mu \mathrm{g} / \mathrm{L})$ & 537 & 1,00 & Référence & 537 & 1,00 & Référence \\
\hline Quartile $2(0,7$ à $1,2 \mu \mathrm{g} / \mathrm{L})$ & 479 & 1,41 & 0,88 à 2,26 & 478 & 1,27 & 0,80 à 2,02 \\
\hline Quartile 3 (1,3 à 2,4 $\mu \mathrm{g} / \mathrm{L})$ & 636 & 1,31 & 0,86 à 1,99 & 634 & 1,32 & 0,89 à 1,98 \\
\hline Quartile 4 (> 2,4 $\mu \mathrm{g} / \mathrm{L})$ & 618 & 1,41 & 0,78 à 2,55 & 618 & 1,28 & 0,72 à 2,27 \\
\hline Valeur $p$ pour la tendance ${ }^{d}$ & \multicolumn{3}{|c|}{0,434} & & \multicolumn{2}{|c|}{0,616} \\
\hline Log BPA (variable continue) & 2270 & 1,08 & 0,92 à 1,28 & 2267 & 1,07 & 0,91 à 1,26 \\
\hline \multicolumn{7}{|l|}{ Femmes } \\
\hline Quartile $1(<0,7 \mu \mathrm{g} / \mathrm{L})$ & 805 & 1,00 & Référence & 802 & 1,00 & Référence \\
\hline Quartile 2 (0,7 à $1,2 \mu \mathrm{g} / \mathrm{L})$ & 524 & 0,95 & 0,55 à 1,65 & 523 & 1,01 & 0,57 à 1,77 \\
\hline Quartile 3 (1,3 à 2,4 $\mu \mathrm{g} / \mathrm{L})$ & 575 & 0,76 & 0,43 à 1,36 & 575 & 0,80 & 0,45 à 1,44 \\
\hline Quartile 4 (> 2,4 $\mu \mathrm{g} / \mathrm{L})$ & 538 & 1,00 & 0,60 à 1,66 & 537 & 1,03 & 0,64 à 1,65 \\
\hline Valeur $p$ pour la tendance ${ }^{d}$ & \multicolumn{3}{|c|}{0,902} & & \multicolumn{2}{|c|}{0,864} \\
\hline Log BPA (variable continue) & 2442 & 1,03 & 0,87 à 1,18 & 2437 & 1,03 & 0,90 à 1,18 \\
\hline
\end{tabular}

Abréviations : BPA, bisphénol A; IC, intervalle de confiance; RC, rapport de cotes.

${ }^{\text {a }}$ Tour de taille de $102 \mathrm{~cm}$ ou plus chez les hommes et de $88 \mathrm{~cm}$ ou plus chez les femmes.

${ }^{\mathrm{b}}$ Ajusté en fonction du sexe (modèle global seulement), de l'âge et de la créatinurie.

'Ajusté en fonction des covariables du modèle 1 ainsi que de l'origine ethnique, de la consommation d'alcool, de la consommation de malbouffe et de la consommation de boissons sucrées.

${ }^{\text {d }}$ Valeur $p$ du test de tendance, calculée par modélisation de la médiane de chaque quartile de BPA comme une variable continue.

quartile $4(2,73 \mathrm{~cm}$, IC à $95 \%:-0,14$ à 5,60). Par ailleurs, chaque augmentation $\mathrm{du}$ log naturel de la concentration urinaire de BPA a été associée à une hausse de $0,33 \mathrm{~kg} / \mathrm{m}^{2}$ (IC à $95 \%: 0,10$ à 0,57 ) de l'IMC et à une hausse de $1,00 \mathrm{~cm}$ (IC à $95 \%$ : 0,34 à 1,65 ) du tour de taille (tableau 4). Les associations se sont révélées légèrement plus marquées chez les femmes (valeur $p$ pour l'interaction $>0,05$; données non présentées).

Dans nos analyses de sensibilité, l'inclusion de l'état de jeûne et du moment de collecte des urines n'a pas entraîné de changement supérieur à $10 \%$ dans quelque modèle que ce soit, ce qui signifie que ces variables n'ont eu aucun effet de confusion sur les valeur $p$ pour la tendance $=0,018$ ) que dans l'échantillon total. De même, les résultats n'ont pas changé de façon notoire lorsque les analyses ont été limitées aux participants exempts de diverses maladies chroniques. Enfin, lorsque les analyses stratifiées selon le sexe ont été reconduites en utilisant les quartiles propres à chaque sexe au lieu des quartiles de l'échantillon total, les estimations de l'effet ont légèrement varié (le RC pour l'obésité a été réduit pour les deux sexes dans le cas du quartile 4 par rapport au quartile 1) mais sont généralement allées dans la même direction, avec des associations positives systématiquement plus fortes chez les hommes.

\section{Analyse}

Notre étude, fondée sur les données de l'ECMS, est la première à porter sur les associations entre le BPA et les indicateurs de l'obésité pour l'ensemble de la population adulte du Canada. Nous avons observé que la concentration urinaire de BPA est positivement associée à l'obésité globale mesurée à partir l'IMC, et ce, de manière dépendante à la dose, et que cette association persiste après ajustement en fonction de divers facteurs potentiels de confusion, dont certains liés à l'alimentation. Ces constatations concordent avec les résultats d'autres études transversales de grande envergure menées auprès d'adultes aux États-Unis $^{11,12}$ et en Chine $^{13}$ ainsi que d'études sur les enfants ${ }^{30-33}$. Les effets observés dans notre étude se sont révélés légèrement moins importants que ceux de l'enquête américaine NHANES (National Health and Nutrition Examination Survey) ${ }^{11}$ (RC [quartile 4 par rapport au quartile 1] $=1,76$ ), se rapprochant davantage de ceux relevés dans l'étude sur des adultes chinois $^{13}$ (RC [quartile 4 par rapport au quartile 1] $=1,50$ ). L'importance moindre de ces associations par rapport à celles des résultats américains pourrait s'expliquer par les concentrations considérablement inférieures de BPA enregistrées dans notre étude et dans l'étude chinoise. Par ailleurs, alors que nos résultats ont plutôt révélé une probabilité croissante d'obésité, certaines études ont fait ressortir un effet seuil ou non monotone d'un quartile de BPA urinaire à l'autre ${ }^{11,15,32}$. Malgré des associations non monotones couramment observées à l'égard du BPA et d'autres perturbateurs endocriniens dans des études toxicologiques $^{34}$, les expositions fréquentes de faible niveau au BPA chez l'humain constituent un défi pour l'évaluation et l'interprétation des relations dose-réponse ${ }^{35}$.
À l'instar des études antérieures ${ }^{11,13,14}$, notre étude fournit des données probantes en faveur de l'existence d'associations positives entre la concentration urinaire de BPA et les mesures de l'IMC et du tour de taille. Cependant, contrairement aux études menées aux États-Unis ${ }^{11,12}$ et en Asie $^{13,14}$, la nôtre n'a pas permis d'établir d'association entre la concentration urinaire de BPA et l'obésité abdominale fondée sur des valeurs 


\section{FIGURE 1}

Différence moyenne entre (A) l'indice de masse corporelle et (B) le tour de taille dans les quartiles de concentration urinaire de bisphénol $A$ (par rapport au quartile 1 ) chez les adultes de 18 à 79 ans, globalement et par sexe, Enquête canadienne sur les mesures de la santé, 2007-2011

A - Indice de masse corporelle

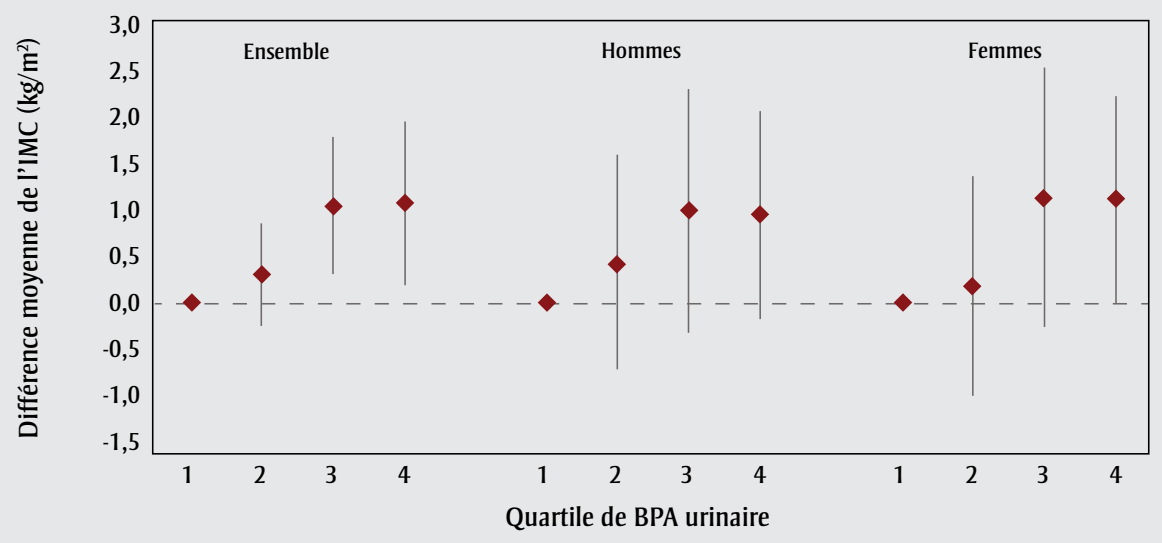

B - Tour de taille

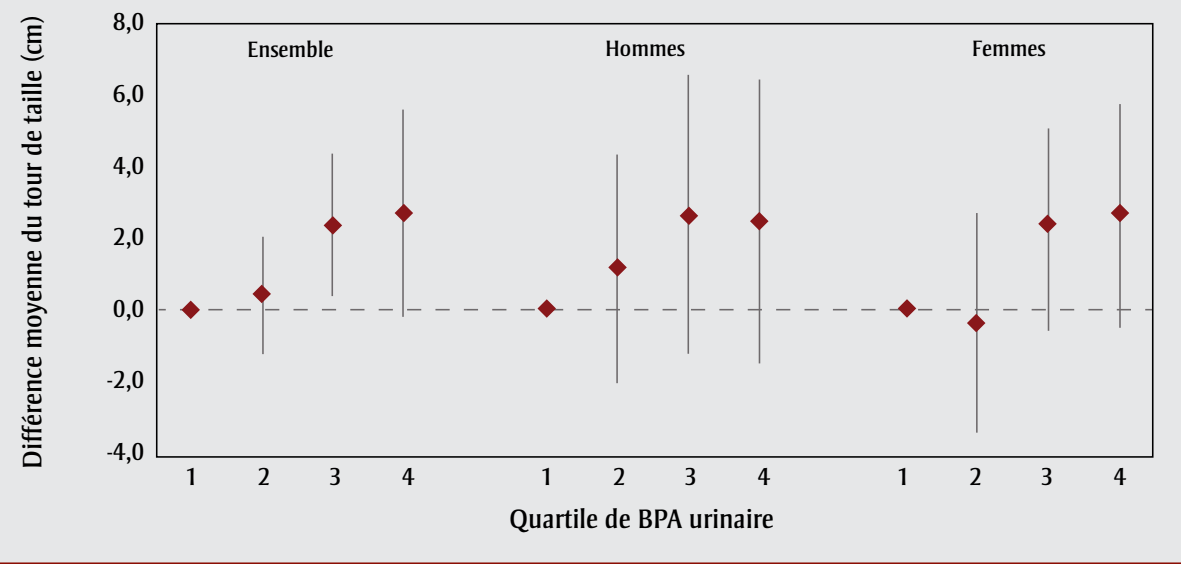

Abréviations : BPA, bisphénol A; IMC, indice de masse corporelle.

Remarques : Quartile 1 : moins de $0,7 \mu \mathrm{g} / \mathrm{L}$; quartile $2: 0,7$ à 1,2 $\mu \mathrm{g} / \mathrm{L}$; quartile $3: 1,3$ à 2,4 $\mu \mathrm{g} / \mathrm{L}$; quartile $4:$ plus de $2,4 \mu \mathrm{g} / \mathrm{L}$.

Tous les modèles ont été ajustés en fonction du sexe (modèles globaux seulement), de l'âge, de la créatinurie, de l'origine ethnique, de la consommation d'alcool et de la consommation de malbouffe. Les modèles du tour de taille ont de plus été ajustés en fonction de la consommation de boissons sucrées. Les barres d'erreur correspondent aux intervalles de confiance à $95 \%$.

seuils du tour de taille. Bien que notre étude ait utilisé les mêmes valeurs seuils que l'étude américaine, les différences entre les populations sur le plan de l'origine ethnique pourraient avoir contribué à ces constatations divergentes $^{36}$ : la concentration urinaire de BPA peut varier selon l'origine ethnique et s'est révélée significativement plus élevée chez les AfroAméricains que chez les Blancs américains ${ }^{27}$. Cependant, en raison de la faible taille des échantillons divisés en groupes ethniques, nous n'avons pas pu faire d'ajustements en fonction de l'origine ethnique ni examiner les associations de ce point de vue.

Plusieurs mécanismes biologiques ont été proposés pour expliquer l'association entre le BPA et l'obésité, mais leur mode d'action exact demeure inconnu. Par exemple, on sait que le BPA favorise la différenciation des adipocytes et l'accumulation de tissus adipeux $^{9}$, qu'il se fixe aux récepteurs œstrogéniques des adipocytes et qu'il inhibe la production de l'hormone adiponectine $^{37}$. Alors que la plupart des études chez l'animal ont porté sur l'exposition in utero, Miyawaki et ses collaborateurs ${ }^{10}$ ont révélé que l'exposition au BPA durant les périodes périnatale et postnatale avait entraîné des gains de poids chez la souris. De plus, l'exposition de longue durée au BPA a accru la masse adipeuse et induit une résistance à l'insuline, une hyperglycémie et une hypercholestérolémie chez des souris adultes ${ }^{38}$, ce qui conduit à penser que le BPA intervient dans l'obésité et dans les problèmes cardiométaboliques. De même, les études épidémiologiques produisent de plus en plus de données probantes indiquant que l'exposition au BPA non seulement favorise l'obésité mais pourrait être associée à un risque accru de diabète et de maladie cardiovasculaire ${ }^{39}$.

Conformément à la plupart des études épidémiologiques, nous n'avons décelé aucune preuve que le sexe ait une incidence sur l'association entre la concentration urinaire de BPA et l'obésité. Des différences entre les sexes en ce qui concerne l'association entre le BPA et l'obésité ont cependant été notées dans des études chez l'animal ${ }^{16,40}$, peut-être en raison de différences sur le plan du métabolisme du BPA $^{41}$ et de l'expression des récepteurs œstrogéniques ${ }^{42}$ ou encore d'effets du BPA spécifiques à un sexe en matière d'apport alimentaire et de dépense énergétique ${ }^{43}$. Cette plausibilité biologique nécessite des recherches plus poussées pour définir les différences possibles selon le sexe dans les résultats de santé associés au BPA.

\section{Points forts et limites}

Notre étude était la première au Canada à porter sur les associations entre le BPA et les indicateurs de l'obésité chez les adultes. Ses points forts sont le fait qu'elle ait été fondée sur la population, la grande taille de son échantillon, l'utilisation de mesures anthropométriques directes, la haute qualité des dosages du BPA dans l'urine et la prise en compte de multiples facteurs potentiels de confusion, dont plusieurs liés à l'alimentation. Ce dernier point est important, car des revues systématiques récentes ont révélé que l'absence d'ajustement en fonction de l'alimentation, et particulièrement de la consommation d'aliments transformés, constituait une limite importante des études sur les associations entre le BPA et divers résultats de santée ${ }^{26,35,39}$.

Notre étude comportait aussi quelques limites. D'abord, en raison de la nature transversale des données de l'ECMS, aucune relation temporelle n'a pu être établie. Il est possible que les personnes obèses accumulent, métabolisent ou éliminent le BPA différemment des autres personnes et que cela explique les concentrations plus élevées de BPA dans leur urine $^{44,45}$. Cela dit, et bien que d'autres études longitudinales soient nécessaires pour les deux sexes, on a pu associer, dans 
TABLEAU 4

Associations entre la concentration urinaire de bisphénol A transformée en log naturel et les mesures de l'indice de masse corporelle et du tour de taille chez les adultes de 18 à 79 ans, globalement et par sexe, Enquête canadienne sur les mesures de la santé, 2007-2011

\begin{tabular}{|c|c|c|c|c|c|c|}
\hline & \multicolumn{3}{|c|}{$\begin{array}{l}\text { Indice de masse corporelle } \\
\qquad\left(\mathbf{k g} / \mathrm{m}^{2}\right)^{\mathrm{a}}\end{array}$} & \multicolumn{3}{|c|}{ Tour de taille $(\mathrm{cm})^{\mathrm{b}}$} \\
\hline & $\mathbf{N}$ & $\beta^{c}$ & IC à 95 \% & $\mathbf{N}$ & $\beta^{c}$ & IC à $95 \%$ \\
\hline \multicolumn{7}{|l|}{ Échantillon total } \\
\hline Log BPA (variable continue) & 4715 & 0,33 & 0,10 à 0,57 & 4704 & 1,00 & 0,34 à 1,65 \\
\hline \multicolumn{7}{|l|}{ Hommes } \\
\hline Log BPA (variable continue) & 2271 & 0,28 & $-0,04$ à 0,59 & 2267 & 0,72 & $-0,22$ à 1,67 \\
\hline \multicolumn{7}{|l|}{ Femmes } \\
\hline Log BPA (variable continue) & 2444 & 0,38 & 0,73 à 2,28 & 2437 & 1,16 & 0,39 à 1,92 \\
\hline
\end{tabular}

Abréviations : BPA, bisphénol A; IC, intervalle de confiance.

${ }^{a}$ Ajusté en fonction du sexe (modèle global seulement), de l'âge, de la créatinurie, de l'origine ethnique, de la consommation d'alcool et de la consommation de malbouffe.

${ }^{\mathrm{b}}$ Ajusté en fonction du sexe (modèle global seulement), de l'âge, de la créatinurie, de l'origine ethnique, de la consommation d'alcool, de la consommation de malbouffe et de la consommation de boissons sucrées.

' Modification moyenne de l'indice de masse corporelle $\left(\mathrm{kg} / \mathrm{m}^{2}\right)$ ou du tour de taille $(\mathrm{cm})$ par unité d'augmentation du log naturel de la concentration urinaire de BPA.

une étude de cohorte prospective menée récemment sur un groupe de femmes, des concentrations urinaires de BPA plus élevées au départ et une prise de poids plus importante sur une période de suivi de 10 ans $^{46}$. De plus, les échantillons ponctuels d'urine pourraient ne pas être représentatifs d'une exposition de longue durée au BPA. Une variabilité intra-individuelle considérable de la concentration urinaire de BPA a en effet été constatée au fil de la journée et de la semaine ${ }^{47}$ ainsi que sur une période de 1 à 3 ans $^{48}$, traduisant vraisemblablement les variations de l'apport alimentaire. Nos analyses de sensibilité ont quant à elles montré que le moment de la collecte des urines et l'état de jeûne n'avaient pas influé sur les associations observées. De plus, dans une étude de groupe récente où plusieurs mesures du BPA urinaire et de l'IMC ont été faites sur une période de 3 ans, des associations positives significatives avec le surpoids ont été établies chez les personnes âgées, et ce, que les concentrations de BPA analysées aient été ponctuelles ou fondées sur des moyennes ${ }^{15}$.

\section{Conclusion}

Nous avons montré que, dans un échantillon national représentatif des adultes canadiens, le BPA était positivement associé à l'obésité en général de même qu'aux valeurs de l'IMC et du tour de taille. Bien que le déséquilibre entre l'apport et la dépense énergétiques demeure un facteur qui contribue grandement à l'obésité, les conséquences attribuables au BPA sur la santé et sur l'économie ne devraient pas être ignorées ${ }^{49}$. De plus, comme le BPA n'est pas interdit au Canada sauf dans les biberons pour bébés et les produits cosmétiques, il est recommandé d'en limiter l'exposition par les emballages alimentaires en raison de ses effets possibles sur la santé, dont l'obésité ${ }^{6}$. Il est nécessaire d'explorer davantage le potentiel du BPA comme environnement obésogène. Des études plus poussées devraient viser à produire des données prospectives et des mesures sur de longues périodes pour améliorer la classification de l'exposition et éclaircir la relation temporelle entre le BPA et l'obésité.

\section{Remerciements}

L'ECMS a été menée par Statistique Canada en partenariat avec Santé Canada et l'Agence de la santé publique du Canada, grâce à l'appui financier du gouvernement fédéral du Canada. Aucun financement externe n'a été obtenu pour cette étude.

\section{Conflits d'intérêts}

Les auteurs déclarent n'avoir aucun conflit d'intérêts.

\section{Contributions des auteurs et avis}

MTD et VCC ont imaginé l'étude, effectué les analyses de données, interprété les données et rédigé l'ébauche du manuscrit. MAM et MdG ont contribué à l'interprétation des données, fourni une expertise et des conseils sur des questions précises et assuré la révision et l'examen critique du manuscrit.

Le contenu de cet article et les opinions qui y sont exprimées n'engagent que les auteurs et ne représentent pas forcément les opinions du Gouvernement du Canada.

\section{Références}

1. Jia H, Lubetkin EI. Trends in qualityadjusted life-years lost contributed by smoking and obesity. Am J Prev Med. 2010;38(2):138-144.

2. Manuel DG, Tuna M, Hennessy D, et al. Projections of preventable risks for cardiovascular disease in Canada to 2021: a microsimulation modelling approach. CMAJ Open. 2014;2(2): E94-101.

3. Bancej C, Jayabalasingham B, Wall RW, et al. Synthèse portant sur les données probantes - Tendances et projections relatives à l'obésité chez les Canadiens. Promotion de la santé et prévention des maladies chroniques au Canada. 2015;35(7):116-119.

4. Organisation mondiale de la santé. Obésité : prévention et prise en charge de l'épidémie mondiale : rapport d'une consultation de l'OMS. Genève (Suisse) : Organisation mondiale de la santé; 2003.

5. Baillie-Hamilton PF. Chemical toxins: a hypothesis to explain the global obesity epidemic. J Altern Complement Med. 2002;8(2):185-192.

6. Santé Canada. Deuxième rapport sur la biosurveillance humaine des substances chimiques de l'environnement au Canada: résultats de l'Enquête canadienne sur les mesures de la santé, cycle 2 (2009 à 2011). Ottawa (Ont.) : Santé Canada; 2013.

7. Bushnik T, Haines D, Levallois $P$, Levesque J, Van Oostdam J, Viau C. Concentrations de plomb et de bisphénol A pour la population canadienne. Rapports sur la santé. 2010;21(3):7-18. 
8. Calafat AM, Ye X, Wong LY, Reidy JA, Needham LL. Exposure of the U.S. population to bisphenol A and 4-tertiary-octylphenol: 2003-2004. Environ Health Perspect. 2008;116(1):39-44.

9. Masuno $\mathrm{H}$, Iwanami J, Kidani $\mathrm{T}$, Sakayama K, Honda K. Bisphenol A accelerates terminal differentiation of 3T3-L1 cells into adipocytes through the phosphatidylinositol 3-kinase pathway. Toxicol Sci. 2005;84(2):319-327.

10. Miyawaki J, Sakayama K, Kato H, Yamamoto $\mathrm{H}$, Masuno $\mathrm{H}$. Perinatal and postnatal exposure to bisphenol A increases adipose tissue mass and serum cholesterol level in mice. J Atheroscler Thromb. 2007;14(5):245-252.

11. Carwile JL, Michels KB. Urinary bisphenol A and obesity: NHANES 2003-2006. Environ Res. 2011;111(6): $825-830$

12. Shankar A, Teppala S, Sabanayagam C. Urinary bisphenol A levels and measures of obesity: results from the National Health and Nutrition Examination Survey 2003-2008. ISRN Endocrinol. 2012;2012:965243.

13. Wang T, Li M, Chen B, et al. Urinary bisphenol A (BPA) concentration associates with obesity and insulin resistance. J Clin Endocrinol Metab. 2012; 97(2):E223-E227.

14. Ko A, Hwang MS, Park JH, Kang HS, Lee HS, Hong JH. Association between urinary bisphenol A and waist circumference in Korean adults. Toxicol Res. 2014;30(1):39-44.

15. Lee MR, Kim JH, Choi YH, Bae S, Park C, Hong YC. Association of bisphenol A exposure with overweight in the elderly: a panel study. Environ Sci Pollut Res Int. 2015;22(12):9370-9377.

16. Caporossi L, Papaleo B. Exposure to bisphenol $\mathrm{A}$ and gender differences: from rodents to human evidences and hypothesis about the health effects. J Xenobiotics. 2015;5(1):5264.

17. Statistique Canada. Guide de l'utilisateur des données de l'Enquête canadienne sur les mesures de la santé (ECMS) : cycle 1. Ottawa (Ont.) : Statistique Canada; 2011.
18. Statistique Canada. Guide de l'utilisateur des données de l'Enquête canadienne sur les mesures de la santé (ECMS) : cycle 2. Ottawa (Ont.) : Statistique Canada; 2013.

19. Statistique Canada. Instructions pour la combinaison des données des cycles 1 et 2 de l'Enquête canadienne sur les mesures de la santé (ECMS). Ottawa (Ont.) : Statistique Canada; 2014.

20. Institut national de santé publique du Québec (INSPQ). Analytical method for the determination of bisphenol A, triclosan, triclocarban and pesticide metabolites in urine by GC-MS-MS (E-454), version condensée pour l'ECMS. Québec (Qué.) : Laboratoire de toxicologie de l' INSPQ; 2009.

21. Institut national de santé publique du Québec (INSPQ). Méthode pour le dosage de la créatinine dans l'urine sur Hitachi 917 (C-530), version condensée. Québec (Qué.) : Laboratoire de toxicologie de l'INSPQ; 2008.

22. Barr DB, Wilder LC, Caudill SP, Gonzalez AJ, Needham LL, Pirkle JL. Urinary creatinine concentrations in the U.S. population: implications for urinary biologic monitoring measurements. Environ Health Perspect. 2005; 113(2):192-200.

23. Société canadienne de physiologie de l'exercice. Guide du conseiller en condition physique et habitudes de vie, 3e éd. Ottawa (Ont.) : Société canadienne de physiologie de l'exercice; 2003.

24. Douketis JD, Paradis G, Keller H, Martineau C. Canadian guidelines for body weight classification in adults: application in clinical practice to screen for overweight and obesity and to assess disease risk. CMAJ. 2005; 172(8):995-998.

25. Mendez MA, Popkin BM, Jakszyn P, et al. Adherence to a Mediterranean diet is associated with reduced 3-year incidence of obesity. J Nutr. 2006;136(11): 2934-2938.

26. LaKind JS, Goodman M, Mattison DR. Bisphenol A and indicators of obesity, glucose metabolism/type 2 diabetes and cardiovascular disease: a systematic review of epidemiologic research Crit Rev Toxicol. 2014;44(2):121-150.
27. LaKind JS, Levesque J, Dumas P, Bryan S, Clarke J, Naiman DQ. Comparing United States and Canadian population exposures from National Biomonitoring Surveys: bisphenol A intake as a case study. J Expo Sci Environ Epidemiol. 2012; 22(3):219-226.

28. Lang IA, Galloway TS, Scarlett A, et al. Association of urinary bisphenol A concentration with medical disorders and laboratory abnormalities in adults. JAMA. 2008;300(11):1303-1310.

29. You L, Zhu X, Shrubsole MJ, et al. Renal function, bisphenol A, and alkylphenols: results from the National Health and Nutrition Examination Survey (NHANES 2003-2006). Environ Health Perspect. 2011;119(4):527-533.

30. Bhandari R, Xiao J, Shankar A. Urinary bisphenol A and obesity in U.S. children. Am J Epidemiol. 2013; 177(11):1263-1270.

31. Eng DS, Lee JM, Gebremariam A, Meeker JD, Peterson K, Padmanabhan $\mathrm{V}$. Bisphenol A and chronic disease risk factors in U.S. children. Pediatrics. 2013;132(3):E637-E645.

32. Trasande L, Attina TM, Blustein J. Association between urinary bisphenol A concentration and obesity prevalence in children and adolescents. JAMA. 2012;308(11):1113-1121.

33. Li DK, Miao M, Zhou Z, et al. Urine bisphenol-A level in relation to obesity and overweight in school-age children. PLoS One. 2013;8(6):E65399.

34. Vandenberg LN. Non-monotonic dose responses in studies of endocrine disrupting chemicals: bisphenol $\mathrm{A}$ as a case study. Dose Response. 2013;12(2): 259-276.

35. Oppeneer SJ, Robien K. Bisphenol A exposure and associations with obesity among adults: a critical review. Public Health Nutr. 2015;18(10): 1847-1863.

36. Lear SA, James PT, Ko GT, Kumanyika S. Appropriateness of waist circumference and waist-to-hip ratio cutoffs for different ethnic groups. Eur J Clin Nutr. 2010;64(1):42-61. 
37. Hugo ER, Brandebourg TD, Woo JG, Loftus J, Alexander JW, Ben-Jonathan N. Bisphenol A at environmentally relevant doses inhibits adiponectin release from human adipose tissue explants and adipocytes. Environ Health Perspect. 2008;116(12):1642-1647.

38. Marmugi A, Lasserre F, Beuzelin D, et al. Adverse effects of long-term exposure to bisphenol A during adulthood leading to hyperglycaemia and hypercholesterolemia in mice. Toxicology. 2014;325:133-143.

39. Rancière F, Lyons JG, Loh VH, et al. Bisphenol A and the risk of cardiometabolic disorders: a systematic review with meta-analysis of the epidemiological evidence. Environ Health. 2015;14:46.

40. vom Saal FS, Nagel SC, Coe BL, Angle $\mathrm{BM}$, Taylor JA. The estrogenic endocrine disrupting chemical bisphenol A (BPA) and obesity. Mol Cell Endocrinol. 2012;354(1-2):74-84.

41. Takeuchi T, Tsutsumi O, Nakamura N, et al. Gender difference in serum bisphenol A levels may be caused by liver UDP-glucuronosyltransferase activity in rats. Biochem Biophys Res Commun. 2004;325(2):549-554.

42. Davis KE, Neinast MD, Sun K, et al. The sexually dimorphic role of adipose and adipocyte estrogen receptors in modulating adipose tissue expansion, inflammation, and fibrosis. Mol Metab. 2013;2(3):227-242.

43. Mackay H, Patterson ZR, Khazall R, Patel S, Tsirlin D, Abizaid A. Organizational effects of perinatal exposure to bisphenol-A and diethylstilbestrol on arcuate nucleus circuitry controlling food intake and energy expenditure in male and female CD-1 mice. Endocrinology. 2013;154(4): 1465-1475.

44. Hays SM, Aylward LL, Blount BC. Variation in urinary flow rates according to demographic characteristics and body mass index in NHANES: potential confounding of associations between health outcomes and urinary biomarker concentrations. Environ Health Perspect. 2015;123(4):293-300.
45. Geens T, Neels H, Covaci A. Distribution of bisphenol-A, triclosan and n-nonylphenol in human adipose tissue, liver and brain. Chemosphere. 2013;87(7):796-802.

46. Song Y, Hauser R, Hu FB, Franke AA, Liu S, Sun Q. Urinary concentrations of bisphenol A and phthalate metabolites and weight change: a prospective investigation in US women. Int J Obes (Lond). 2014;38(12):1532-1537.

47. Ye X, Wong LY, Bishop AM, Calafat AM. Variability of urinary concentrations of bisphenol A in spot samples, first morning voids, and 24-hour collections. Environ Health Perspect. 2011;119(7):983-988.

48. Townsend MK, Franke AA, Li X, Hu FB, Eliassen AH. Within-person reproducibility of urinary bisphenol A and phthalate metabolites over a 1 to 3 year period among women in the Nurses' Health Studies: a prospective cohort study. Environ Health. 2013; 12(1):80.

49. Trasande L. Further limiting bisphenol A in food uses could provide health and economic benefits. Health Aff. 2014;33(2):316-323. 